\title{
Références bibliographiques du dossier « quel avenir pour les études en sciences humaines?»
}

Bernadette Plumelle

\section{OpenEdition}

1 Journals

Édition électronique

URL : http://journals.openedition.org/ries/583

DOI : 10.4000/ries.583

ISSN : 2261-4265

Éditeur

Centre international d'études pédagogiques

Édition imprimée

Date de publication : 1 décembre 2008

Pagination : 133-144

ISSN : 1254-4590

\section{Référence électronique}

Bernadette Plumelle, « Références bibliographiques du dossier « quel avenir pour les études en

sciences humaines ? » », Revue internationale d'éducation de Sèvres [En ligne], 49 | décembre 2008, mis en ligne le 29 juin 2011, consulté le 01 mai 2019. URL : http://journals.openedition.org/ries/583 ; DOI $10.4000 /$ ries.583

(c) Tous droits réservés 


\title{
Références bibliographiques
}

\section{Bernadette Plumelle}

L'avenir des études en sciences sociales et humaines est un champ d'étude peu traité et les publications sur ce sujet ne sont pas très nombreuses, ce qui nous a amenée, pour une bibliographie sur cette question, à élargir notre sélection aux sciences sociales, à l'université et à ses évolutions, à l'accès à l'emploi ainsi qu'à une analyse prospective de l'enseignement supérieur et au rôle que les sciences sociales pourraient jouer.

Nous avons fait le choix de sélectionner principalement des ouvrages, rapports et articles très récents. En introduction, on trouvera une sélection de documents sur l'enseignement supérieur dans le monde et sur ses transformations, notamment avec le Processus de Bologne. La première partie donne un aperçu des débats qui traversent les sciences sociales et humaines (SHS). Les deux parties suivantes rassemblent des références sur les filières de sciences sociales et l'insertion professionnelle des diplômés en SHS, avec un point particulier sur le cas français. Une dernière partie propose des études sur les enjeux liés aux transformations de l'enseignement supérieur et au rôle assigné aux sciences sociales.

Une sitographie complète la bibliographie arrêtée le 22 octobre 2008. Les documents mentionnés sont pour la plupart consultables au centre de ressources documentaires du CIEP ou accessibles en ligne.

\section{L'ENSEIGNEMENT SUPÉRIEUR DANS LE MONDE}

\begin{abstract}
BANQUE MONDIALE, Construire les sociétés du savoir : nouveaux défis pour l'enseignement supérieur, Presses de l'université de Laval/Québec, 2003, 275 p., bibliogr.

Le rapport s'inscrit dans la suite d'un colloque organisé par la Banque mondiale (BM) en 2002 : Higher education in developing countries : Peril and Promise. Il décrit la stratégie de la $\mathrm{BM}$ relative à l'enseignement supérieur et le rôle des universités dans une économie mondiale de plus en plus fondée sur la connaissance. Après un chapitre sur l'environnement mondial et ses transformations, le rapport décrit la crise dont souffre l'enseignement supérieur dans les pays en développement et les pays en transition, et l'évolution de ses rapports avec le marché et l'État.
\end{abstract}

CHARLE Christophe, VERGER Jacques, Histoire des universités, PUF/Paris, 2007, 126 p. (Que sais-je?)

L'université est une institution née en Italie, en France et en Angleterre au début du XIII siècle. Ce modèle s'est ensuite répandu dans toute l'Europe et, à partir du XVIe siècle et particulièrement aux $\mathrm{XIX}^{\mathrm{e}}$ et $\mathrm{XX}^{\mathrm{e}}$ siècles, sur tous les continents. L'ouvrage étudie successivement les différentes périodes de son développement.

DAVIDENKOFF Emmanuel, KAHN Sylvain, Les universités sont-elles solubles dans la mondialisation?, Hachette Littératures/Paris, 2006, 214 p.

Les auteurs analysent les idées reçues et les discours sur la mondialisation de l'enseignement supérieur tenus par leurs détracteurs comme par leurs défenseurs. Avec le Processus de 
Bologne (1999), l'Europe des universités tente de se réformer afin d'affirmer sa compétitivité en matière de recherche et d'innovation mais, selon les auteurs, les résistances au changement sont tenaces. Chaque «idée reçue» fait l'objet d'un chapitre.

DIZAMBOURG Bernard coord., L'enseignement supérieur, une compétition mondiale ? $^{1}$, Revue internationale d'éducation de Sèvres, septembre 2007, $n^{\circ} 45$, $169 p$.

La production et la transmission des connaissances sont aujourd'hui un enjeu de compétition internationale. Les contributions rassemblées dans ce dossier apportent un éclairage sur l'évolution de l'enseignement supérieur dans une dizaine de pays et, plus largement, sur un paysage universitaire en cours de recomposition.

\section{Le Processus de Bologne}

CROSIER David, PURSER Lewis, SMIDT Hanne, Trends V: Universities shaping the european higher education area, European University Association (EAU)/Bruxelles, 2007, 95 p. [rapport en ligne]

Ce rapport s'inscrit dans une série lancée en 1999, et visant à suivre l'évolution des politiques des États européens en matière d'enseignement supérieur et de recherche. Plus de 900 institutions européennes ont contribué à ce cinquième opus. Il porte, entre autres, sur la réforme structurelle; les outils de Bologne pour la mobilité et la reconnaissance des diplômes; l'assurance qualité ou l'apprentissage tout au long de la vie.

EURYDICE : Réseau d'information sur l'éducation en Europe, Focus sur les structures de l'enseignement supérieur en Europe 2006/2007: évolutions nationales dans le cadre du Processus de Bologne, Eurydice/Bruxelles, 2007, 348 p.

Cette publication présente, sous forme de diagramme, les principaux parcours de formation pour l'année 2004-2005, les conditions d'accès aux filières et leurs durées respectives. Chaque diagramme est complété par un état des lieux des réformes ou dispositifs introduits en lien avec le Processus de Bologne. En introduction, le rapport présente un bref historique du Processus de Bologne suivi par une synthèse comparative des principales tendances qui se dégagent au niveau européen.

\section{TUNING PROJECT, Introduction à Tuning, pour une convergence des struc-} tures éducatives en Europe : contribution des universités au Processus de Bologne, décembre 2006, 104 p. [rapport en ligne]

Le projet Tuning, lancé en 2000, rassemble plus d'une centaine d'universités européennes. Il concerne plusieurs lignes d'action définies par le Processus de Bologne et notamment l'adoption d'un système facilitant la lisibilité et la comparabilité des diplômes. Le projet a pour objet la définition des points de référence pour les compétences générales et les compétences spécifiques à une matière, dans une série de disciplines : gestion d'entreprise, sciences de l'éducation, géologie, histoire, mathématiques, physique et chimie. Ce rapport contient les documents principaux élaborés par le projet.

1. La bibliographie de ce numéro recense les travaux dans les thématiques suivantes : internationalisation des enseignements, mouvement de privatisation, importance accrue de la recherche et mise en place de démarches de qualité. On y trouve des études comparatives et des monographies régionales et nationales. La deuxième partie porte sur l'Europe et sur le Processus de Bologne (1999); la dernière partie recense des études et les rapports officiels sur l'enseignement supérieur en France. Ce document est accessible sur le site du CIEP. 
SCHULTHEIS Franz dir., ROCA i ESCODA Marta dir., COUSIN Paul-Frantz dir., Le cauchemar de Humboldt : les réformes de l'enseignement supérieur européen, Raisons d'agir Éditions/Paris, 2008, 231 p.

En juin 1999, les ministres de l'Éducation de 29 États européens signaient la Déclaration de Bologne sur l'harmonisation des systèmes d'enseignement supérieur. Les différents contributeurs apportent un regard critique sur les effets du Processus de Bologne et en évaluent les conséquences, en Allemagne, France, Italie et Suisse. Pour la France, les mutations de la faculté des lettres et sciences humaines sont analysées, de même que «les études littéraires au risque de Bologne» dans le secondaire et le supérieur.

\section{LES SCIENCES SOCIALES AUJOURD'HUI}

Commission Gulbenkian pour la restructuration des sciences sociales présidée par Immanuel Wallerstein, Ouvrir les sciences sociales, 1996, 100 p. [rapport en ligne]

Dix chercheurs d'origines et de disciplines différentes se sont réunis «afin de préparer l'indispensable renouveau des sciences humaines et sociales» et le résultat de leurs travaux se présente comme un manifeste. Le rapport offre, dans une première partie, un examen rétrospectif de l'histoire des sciences sociales avec les dynamiques de constitution et de différenciation des disciplines. Les deux parties suivantes portent sur les débats au sein des sciences sociales depuis 1945 et la structure organisationnelle des sciences sociales. Enfin, des pistes concrètes pour leur reconstruction sont proposées.

FUMAROLI Marc, "Les humanités ou la critique des spécialités» in MICHAUD Y. dir. Qu'est-ce que la culture?, Odile Jacob/Paris, 2001, p. 287-298

Texte de la conférence de Marc Fumaroli prononcée dans le cadre de l'«Université de tous les savoirs".

GODELIER Maurice, L'état Des Sciences de l'Homme et de la Société en France et leur rôle dans la construction de l'espace européen de la recherche, La Documentation française/Paris, 2002, 368 pages [rapport en ligne]

Le rapport présente le paysage des sciences de l'homme et de la société (SHS) en France et en Europe. Après un rappel de l'ancienne distinction entre les sciences humaines, «les humanités», et les sciences sociales, il est question de l'organisation de la recherche en SHS en France; l'auteur analyse ensuite les forces et les faiblesses de ces domaines scientifiques. La dernière partie est consacrée aux mesures nécessaires pour leur développement. En annexe : des études sur plusieurs pays européens.

HALL Stuart, Identités et cultures: politiques des cultural studies, Éditions Amsterdam/Paris, 2008, 411 p.

Recueil d'articles d'une des figures principales du mouvement théorique des cultural studies (CS). Les quatorze textes rassemblés (de 1974 à 2002) sont distribués en quatre parties : la généalogie critique des cultural studies; les fondements théoriques; la redéfinition des notions de «culture» et de "populaire»; l'émergence et le développement de ces cursus face aux disciplines classiques des humanités. 
KAZANCIGIL Ali dir., MAKINSON David dir., Les sciences sociales dans le monde, Éditions UNESCO-Éditions de la Maison des sciences de l'homme/Paris, 2001, 402 p.

À quoi servent les sciences sociales? L'ouvrage, composé d'une quarantaine d'articles, apporte une multiplicité de points de vue sur cette discipline. La première partie fait un point historique et dresse un panorama des sciences sociales dans toutes les parties du monde. La seconde partie aborde de grands domaines (science et technologie, développement, environnement, sciences du comportement) ainsi que leurs applications en sciences sociales. De nombreuses données statistiques et des graphiques complètent les articles.

KAGANCIGIL Ali, "Renforcer le rôle des sciences sociales dans la société : l'initiative mondiale en matière de sciences sociales", Revue internationale des sciences sociales, mars 2003, $n^{\circ} 177$, pp. 425-428

L'initiative mondiale en matière de sciences sociales a été lancée par le Conseil international des sciences sociales, afin de réévaluer le fonctionnement des sciences sociales ainsi que leur rôle et leur position dans la société. Elle est née après une série de conférences internationales de l'OCDE sur le thème «Repenser les sciences sociales».

MACKIEWICZ Wolfgang dir., Positioning Humanities research in the 7th framework programme, Commission européenne/Bruxelles, avril 2007, 118 p. [rapport en ligne]

La Direction générale de la recherche de la Commission européenne a mis en place, en février 2006, un groupe de onze experts sur les humanités, présidé par W. Mackiewicz. Leur rapport final souligne que les sciences humaines peuvent apporter un éclairage important sur les changements politiques, sociaux et culturels en Europe, éclairages essentiels à la réalisation des objectifs de Lisbonne. Il précise l'importance de mieux adapter les outils du septième programme cadre recherche et développement (PCRD) aux sciences humaines et s'achève sur un ensemble de recommandations.

\section{MATTELART Armand, NEVEU Erik, Introduction aux cultural studies, La Découverte/Paris, 2008, 121 p. (Repères)}

Comment le milieu social, l'âge, le genre, l'identité «ethnique» affectent-ils les rapports à la culture? Ce sont là quelques-unes des questions que les cultural studies ont choisi d'inscrire à l'ordre du jour de la recherche dans l'Angleterre des années 1960. L'ouvrage retrace les origines et le développement de ce courant de recherche qui renouvelle le débat sur les rapports entre culture et société et bouscule les frontières entre disciplines académiques. Il présente ses grands textes, relate l'internationalisation de ce courant de recherche, analyse ses apports et ses limites.

\section{MESURE Sylvie dir., SAVIDAN Patrick dir., Dictionnaire des sciences humaines, Presses universitaires de France/Paris, 2006, 1275 p. (Quadrige Dicos poche)}

Face à une réalité sociale qui résiste toujours davantage aux grilles d'analyse traditionnelle, ce dictionnaire a pour objet le décryptage du monde contemporain. Plus de trois cent cinquante auteurs, français et étrangers, issus des différentes disciplines des sciences humaines (anthropologie, sociologie, psychologie, psychanalyse, droit, économie, linguistique, histoire, géographie, démographie, science politique, philosophie) ont contribué à la rédaction de cet ouvrage. Les 565 articles, monographies, essais ou synthèses reflètent les orientations, les enjeux mais aussi la fécondité des travaux actuels en sciences humaines. 
PELUS-KAPLAN Marie-Louise dir., Unité et globalité de l'homme : des humanités aux sciences humaines, Syllepse/Paris, 2006, 256 p., (Histoire : enjeux et débats) Issues du tronc commun des «humanités» traditionnelles, les sciences de l'homme se constituent en disciplines à partir du XIX ${ }^{\mathrm{e}}$ siècle par la délimitation progressive de leurs territoires respectifs. Ce volume, dans la continuité d'un colloque organisé en 2002 à Paris, rassemble les contributions de quinze spécialistes, organisées selon trois axes : genèse et métamorphoses des sciences humaines; constitution des sciences humaines en disciplines ( $\mathrm{XIX}^{\mathrm{e}}$ et $\mathrm{XX}^{\mathrm{e}}$ siècles); enfin, les sciences humaines à l'épreuve de la mondialisation.

WIEVIORKA Michel dir., Les sciences sociales en mutation, Éditions Sciences Humaines/Paris, 2007, 624 p.

L'ouvrage, issu d'un colloque organisé à Paris en 2006, rassemble une cinquantaine de contributions de spécialistes du monde entier sur les mutations en cours dans les sciences humaines et sociales (SHS). Il s'organise autour de trois types de questions : sur les outils d'analyse, sur les enjeux actuels et futurs de la recherche en SHS, sur les relations avec les sciences dites «exactes» et celles de la cognition. En introduction, l'auteur apporte un regard historique sur les SHS dans les années soixante jusqu'à la situation actuelle. Danièle Hervieu-Léger examine les conditions dans lesquelles les sciences sociales envisagent leurs relations avec les autres sciences, en premier lieu les sciences expérimentales.

\title{
FILIÈRES DES SCIENCES HUMAINES ET SOCIALES
}

\begin{abstract}
DURAND Béatrice, NEUBERT Stéfanie, RÖSEBERG Dorothée et al., Étudier en France et en Allemagne : approche comparée des cultures universitaires, Presses universitaires du Septentrion/Villeneuve d'Ascq, 2007, 138 p. (Guides pratiques)

Le Processus de Bologne a entrainé un large mouvement d'harmonisation des structures universitaires en Europe, mais les traditions universitaires sont différentes d'un pays à l'autre. Dans cet ouvrage, les auteurs comparent, point par point, les cultures universitaires française et allemande et montrent que le découpage des champs du savoir et le statut des disciplines les unes par rapport aux autres ne sont pas identiques dans les deux pays.
\end{abstract}

EURYDICE : Réseau d'information sur l'éducation en Europe, Chiffres clés de l'enseignement supérieur en Europe: édition 2007, Office des publications officielles des Communautés européennes/Luxembourg, 2007, 176 p.

En 2004, l'Union européenne comptait plus de 17 millions d'étudiants. Ce rapport présente soixante-dix indicateurs sur l'enseignement supérieur en Europe : organisation, participation, ressources matérielles et humaines, aides financières, mobilité internationale et diplômés. Il apparaît que les diplômés en «sciences sociales, commerce et droit» sont les plus nombreux : plus de $25 \%$ des diplômés dans tous les pays, à l'exception de l'Allemagne et de la Suède.

OBA Jun, «Les sciences sociales dans les pays de l'OCDE», in KAZANCIGIL Ali dir., MAKINSON David dir., Les sciences sociales dans le monde, Éditions UNESCO-Éditions de la Maison des sciences de l'homme/Paris, 2001, pp. 62-78 L'auteur présente le financement de la recherche en sciences sociales dans les pays de l'OCDE et les ressources humaines qui y sont affectées, plus particulièrement pour l'enseignement supérieur. Le dernier point concerne l'harmonisation et la diffusion des données concernant l'organisation des sciences sociales. Cette étude fournit plusieurs tableaux comme la part des sciences sociales dans les diplômes universitaires ou le nombre de diplômés universitaires dans ces disciplines. 
OCDE : Organisation de coopération et de développement économiques, Regards sur l'éducation 2008 : les indicateurs de l'OCDE, OCDE/Paris, 2008, 547 p.

Ce rapport annuel présente un ensemble d'indicateurs actualisés et comparables sur les résultats des systèmes d'enseignement des pays de l'OCDE. Cette édition comprend des éléments nouveaux concernant l'enseignement supérieur (taux d'accès, gouvernance, etc.); il comporte un panorama des taux d'accès à l'enseignement tertiaire selon le domaine d'études (p. 62) et la répartition par domaine d'études des titulaires d'un diplôme de l'enseignement supérieur (p. 46).

WORLD BANK, The road not travelled : education reform in the Middle East and North Africa, World Bank/Washington, 2008, 359 p., (MENA development report)

Le rapport de la Banque mondiale couvre tous les niveaux d'instruction formelle et informelle de cette région. Pour ce qui concerne l'enseignement supérieur, les pays ont, dans leur ensemble, multiplié par cinq le nombre d'inscriptions à l'enseignement supérieur et, dans plus de la moitié des pays, environ deux tiers des étudiants se spécialisent dans les sciences sociales et humaines. Mais le taux de chômage des diplômés y est particulièrement élevé.

\section{Les études de sciences sociales en France}

FRYDEL Yves, PAPON Sylvain, Les étudiants inscrits dans les universités publiques françaises en 2007, Note d'information - DEPP, août 2008, 6 p.

138 L'étude porte sur l'analyse statistique des effectifs universitaires (année 2007-2008) des 82 universités publiques françaises. Le nombre d'étudiants diminue pour la deuxième année consécutive, et ceci pour les trois cursus. La baisse est particulièrement marquée en cursus licence et les filières générales sont les plus touchées par cette baisse $(-8,1 \%$ en sciences humaines et sociales).

GOULARD François dir., L'enseignement supérieur en France : état des lieux et propositions, Ministère de l'éducation nationale, de l'enseignement supérieur et de la Recherche/Paris, 2007, 150 p. [rapport en ligne]

Ce rapport établit un état des lieux critique et prospectif de l'enseignement supérieur français. Il présente l'offre française de formation supérieure, l'employabilité et l'insertion des diplômés, ainsi qu'une analyse de la diversité des filières de formation.

THEULIERE Maël, L'évolution des effectifs de l'enseignement supérieur (19902001), Éducation et formations, mars 2004, $n^{\circ} 67$, p. 7-19

La croissance des effectifs inscrits dans l'enseignement supérieur s'est prolongée jusqu'au milieu des années 1990. Avant 1995, la hausse des effectifs se répartissait équitablement entre les trois principales filières (université, formations courtes, grandes écoles); il y a eu ensuite une désaffection des nouveaux bacheliers pour l'université au profit des autres filières. L'auteur analyse les évolutions selon les disciplines (pp. 10-11).

Universités : vers quelle autonomie?, Esprit, décembre 2007, $n^{\circ} 340$, pp. 9-218 Le dossier s'intéresse à la manière dont l'université française s'organise et aux évolutions récentes qu'elle a connues avec, en introduction, les données chiffrées commentées sur le monde étudiant. La première partie porte sur les dynamiques en cours, et plus particulièrement la gouvernance des universités et le rôle des présidents d'université. La seconde partie inscrit l'enseignement supérieur dans le cadre plus large de la société de connaissance. 
VASCONCELLOS Maria, L'enseignement supérieur en France, La Découverte/ Paris, 2006, 121 p. (Repères)

L'ouvrage présente l'enseignement supérieur français et les grands regroupements institutionnels qu'il comporte avec, d'un côté, les grandes écoles et les écoles professionnelles relevant de filières sélectives et, de l'autre, les universités composées essentiellement de filières ouvertes à tous les bacheliers. Les derniers chapitres sont consacrés aux filières des universités dont l'offre de formation s'est transformée profondément sous la pression du nombre et des nouvelles attentes des étudiants.

\title{
Articulation enseignement secondaire / enseignement supérieur
}

\begin{abstract}
CHARVET Pascal coord., DIZAMBOURG Bernard coord., Entre lycée et enseignement supérieur, Revue de l'Inspection générale (La), octobre 2007, 77 p.

Les contributeurs identifient des différences ou des convergences de perspectives, de pratiques et de problématiques entre les cycles secondaire et supérieur. Trois axes ont été privilégiés : les contenus d'enseignement et la conception que l'on se fait des disciplines scolaires et universitaires; les acteurs; les enjeux dont celui de la place du concept de compétences dans les programmes de formation.
\end{abstract}

CONVERT Bernard, "Des hiérarchies maintenues: espace des disciplines, morphologie de l'offre scolaire et choix d'orientation en France, 1987-2001", Actes de la recherche en sciences sociales, 2003, $n^{\circ} 149$, pp. 61-73

L'enseignement français, secondaire et supérieur, a connu de profonds changements dans les dernières décennies, avec une augmentation très forte du nombre de lycéens et une diffusion géographique de l'enseignement universitaire sur l'ensemble du territoire. L'auteur mesure, pour une académie, les effets de ces transformations sur l'accès des différentes catégories d'étudiants aux filières de l'enseignement secondaire et supérieur.

TAVOILLOT Pierre-Henri, "La transmission des savoirs : la culture introuvable?" Les Cahiers français, $n^{\circ}$ 344, mai-juin 2008, pp. 84-89

Après un rappel sur le lien entre culture démocratique et culture générale, l'auteur retrace une histoire de la «culture française» dans le système éducatif français. L'article se termine par des propositions de contenus d'enseignement pour la secondaire comme pour le premier cycle du supérieur.

\section{LES ÉTUDES EN SCIENCES SOCIALES ET L'EMPLOI}

\begin{abstract}
ALLEN Jim ed., VAN DER VELDEN Rolf ed., Flexible Professionnel in the Knowledge Society : general results of the REFLEX project, Research centre for education and the Labour Market: Maastricht university, 2007, 286 p. [rapport en ligne]

Financé par la Commission européenne, ce rapport est le résultat de trois ans d'enquête auprès de 30000 étudiants interrogés en 2005, cinq ans après leur sortie de l'enseignement supérieur. Onze pays européens y ont participé. L'enquête s'articule autour de quatre interrogations : quelles compétences les étudiants de l'enseignement supérieur recherchent-ils pour s'intégrer sur le marché du travail? Quelles sont celles qui sont acquises et celles qui sont requises par
\end{abstract}


les employeurs? Comment les établissements de l'enseignement supérieur les aident-ils à les développer? Et, enfin, quelles sont les tensions en jeu entre les étudiants, les établissements et les employeurs? Cette enquête montre que les étudiants ont globalement une bonne perception de leur formation mais que, d'un pays à l'autre, les écarts entre leur formation d'origine et les compétences exercées ou exigées pour un premier emploi sont très variables.

AURIAT Nadia, "Quelques tendances de l'éducation et de l'emploi dans le domaine des sciences sociales» in KAZANCIGIL Ali dir., MAKINSON David dir., Les sciences sociales dans le monde, Éditions UNESCO-Éditions de la Maison des sciences de l'homme/Paris, 2001, pp. 79-89

L'étude présente quelques tendances principales des activités des sciences sociales dans différentes parties du monde. On y trouve une série de graphiques et de tableaux commentés sur les inscriptions à l'université dans ces filières, la mobilité et l'emploi des étudiants étrangers, les inscriptions des étudiants étrangers et le taux d'installation de ceux-ci dans les pays d'étude.

FAUCHEUX Michel, FOREST Joëlle, CHARDEL Pierre-Antoine et al., Les recherches en sciences humaines et sociales dans les écoles d'ingénieurs, Éditions Petra, 2007 (Acta Stoüca)

Cet ouvrage vise à parcourir l'espace des recherches en sciences humaines et sociales dans les écoles d'ingénieurs. Certaines font l'objet d'une étude spécifique : Grande école des Télécoms, INSA de Strasbourg, École des Mines de Paris. D'autres chapitres sont plus généraux; ils concernent des éléments de méthode, des dispositifs et des pratiques d'interdisciplinarités en écoles d'ingénieurs ou l'analyse des entraves au développement des recherches en SHS dans les écoles d'ingénieurs.

LESSARD Claude éd., ALTET Marguerite éd., PAQUAY Léopold éd. et al., Entre sens commun et sciences humaines : quels savoirs pour enseigner?, De Boeck Université/Bruxelles, 2004, 277 p. (Perspectives en éducation et formation)

L'ouvrage fait le point sur la place des sciences humaines et sociales (SHS) dans le cursus de formation des maitres comme dans l'expertise et les savoirs professionnels des enseignants.

MORA José-Gines, GARCIA-ARACIL Adela, CAROT José-Miguel et al., "Rémunérations monétaires et compétences des jeunes diplômés européens", Politiques et gestion de l'enseignement supérieur, janvier 2006, vol. 18, $n^{\circ} 1$, pp. 43-59, bibliogr., stat.

À partir de données portant sur un échantillon de diplômés européens de l'enseignement supérieur en début de carrière professionnelle (36 000 diplômés du premier cycle de l'enseignement supérieur), les auteurs ont recensé les déterminants des compétences en termes de capital humain (talents, qualifications et capacités) acquises par les jeunes diplômés de l'enseignement supérieur et ceux des compétences requises pour les emplois qu'ils occupent.

PAUL Jean-Jacques, MURDOCH Jaken, «L'enseignement supérieur européen au regard de la préparation professionnelle de ses étudiants", Politiques d'éducation et de formation, février 2001, $n^{\circ} 2, p .49-61$

Les auteurs analysent les conditions d'entrée des étudiants sur le marché du travail et le type d'emploi occupé. Ils s'appuient sur une recherche, le projet CHEERS (Careers after Higher Education : a Européean Research Survey) qui couvre onze pays européens et le Japon. Au sein des différents pays, les études médicales et d'ingénieur conduisent à une situation favorable en terme d'éventail d'emplois; à l'inverse, les sciences humaines et les lettres conduisent fréquemment à des emplois peu spécifiques et ne nécessitant pas toujours un niveau d'études supérieures. 
PERRENOUD Philippe dir., ALTET Marguerite dir., LESSARD Claude dir. et al., Conflits de savoirs en formation des enseignants, De Boeck Université/Bruxelles, 2008, 274 p., bibliogr.

Les différents contributeurs abordent un problème central de la formation des enseignants : les rapports difficiles entre savoirs des praticiens et savoirs issus de la recherche. Un chapitre est consacré aux rapports entre savoirs théoriques (sciences sociales) et savoirs d'expérience dans les instituts de formation, distinguant les curricula selon la vision de la formation initiale qui prévaut et plus particulièrement la conception de l'articulation entre théorie et pratique (pp. 141-153).

\section{L'insertion professionnelle des étudiants en sciences sociales en France}

GIRET Jean-François, BERET Pierre, RECOTILLET Isabelle, "L'évolution des débouchés professionnels des docteurs ", Éducation et formation, 2004, $n^{\circ} 67$, pp 109-116

L'article analyse l'évolution des débouchés professionnels des docteurs depuis le milieu des années 1990 en France. Les conditions de leur insertion professionnelle se sont globalement améliorées, mais les différences entre disciplines se sont accrues, aussi bien à l'intérieur des sciences exactes que des sciences humaines et sociales. La fonction publique apporte moins de débouchés mais les docteurs en SHS trouvent des conditions plus favorables dans la fonction publique. À l'inverse, les docteurs en sciences exactes s'orientent majoritairement vers le secteur privé, avec une meilleure valorisation du point de vue statutaire et salarial.

HERAUT Jean-Alain, "Les diplômés de lettres et sciences humaines et sociales: nouveaux métiers, nouvelles compétences?», Les Dossiers, $n^{\circ}$ 154, juillet 2004

L'hétérogénéité du public étudiant en lettres et sciences humaines et sociales résulte de la massification des études supérieures et de certains biais d'orientation. Cette étude a pour objet les trajectoires professionnelles de ces étudiants, hors l'enseignement et la recherche (débouchés traditionnels de leur discipline), les initiatives et les moyens mis en place par les universités, les compétences développées et les nouveaux métiers exercés.

HETZEL Patrick, De l'Université à l'emploi : rapport final de la Commission du débat national Université-Emploi, La Documentation française, octobre 2006, $112 p$.

Le rapport définit six grandes orientations de nature à mieux relier l'université à l'emploi : lutter contre l'échec à l'université; repenser l'information et l'orientation; améliorer la professionnalisation; rapprocher durablement l'université du monde du travail; créer un partenariat universités/employeurs pour la croissance; faire évoluer l'ensemble du système universitaire.

"Lettres: le défi de la professionnalisation», La Lettre de l'Éducation, 17 décembre 2007, supplément au $n^{\circ}$ 579, 4 p.

Analyse des filières de lettres, sciences humaines et sociales dans les universités françaises : répartition, insertion professionnelle et redéfinition des formations classiques pour une professionnalisation des cursus. 
LETOURNEUX Frédérique, "Les SHS en entreprise : des intérêts mutuels à défricher ", La Lettre de l'Etudiant, octobre 2008, $n^{\circ}$ 920, pp. 4-5

Présentation d'initiatives destinées à rapprocher les jeunes diplômés en SHS et les recruteurs.

"Quelques indicateurs sur l'insertion des jeunes issus de l'enseignement supérieur », Bref, $n^{\circ}$ 253, juin 2008, supplément électronique

Données extraites de l'enquête «Génération 2004» publiée par le CEREQ en 2008.

\section{LES ENJEUX}

Commission des Communautés européennes/Bruxelles, Mobiliser les cerveaux européens : permettre aux universités de contribuer pleinement à la stratégie de Lisbonne : communication de la Commission, 2006, 14 p. [rapport en ligne]

L'Europe veut renforcer tout à la fois l'éducation, la recherche et l'innovation. Le rapport identifie les principaux défis qui attendent l'enseignement supérieur européen : atteindre un niveau de qualité international, améliorer la gouvernance et accroître et diversifier le financement.

DENMAN Brian D., "Comment définir l'université du XXI ${ }^{\mathrm{e}}$ siècle?", Politiques et gestion de l'enseignement supérieur, 2005, vol. 17, $n^{\circ} 2, p p .9-30$

Le terme "université» est très ancien, pourtant sa définition demeure extrêmement controversée. L'article a pour objet de proposer une définition pratique de l'université du XXI siècle. Après une analyse de l'origine historique de l'enseignement supérieur (mobilité des étudiants et transfert de connaissances), l'auteur étudie le contexte actuel et les acteurs de l'université. Il établit un projet de classification des universités, mettant l'accent sur le rôle que ces établissements jouent dans la diffusion et le progrès du savoir par l'enseignement et la recherche.

MATSUURA Kö̈chiro : "Les sciences sociales et humaines jouent un rôle indispensable»(entretien), SHS Regards, mars 2008, pp. 16-17

Le Directeur général de l'UNESCO précise le rôle des sciences sociales et humaines dans la nouvelle stratégie de l'organisation afin, notamment, de développer les dimensions éthiques et sociales de grandes tendances actuelles telles que la mondialisation ou le changement climatique.

JOAS Hans, "Le nouveau rôle des sciences sociales dans la perspective d'une théorie de l'action", La Revue du Mauss, février 2004, n² 24, p. 101-114

Après un diagnostic de la situation des sciences sociales dans le monde, l'auteur ouvre des perspectives de développement de ce champ des sciences.

LAHIRE Bernard dir., ROSENTAL Claude dir., La cognition au prisme des sciences sociales, Éditions des Archives Contemporaines/Paris, 2008, 310 p.

Comment les sciences sociales peuvent-elles contribuer à l'étude de la cognition? Quels objets spécifiques sont-elles à même de construire dans ce domaine à partir des enjeux théoriques et des méthodes qui leur sont propres? Les différents contributeurs, chercheurs en sociologie, en anthropologie, en histoire, en philosophie et en sciences de la communication, examinent les projets de collaboration des sciences sociales avec d'autres disciplines, telles que la psychologie et la neurobiologie. 
OCDE, Tertiary Education for the Knowledge Society, OCDE/Paris, 2008, 2 vol. (324 p. et 390 p.)

Le rapport analyse le développement de l'enseignement supérieur dans vingt-quatre pays. Il montre sa croissance rapide (+ 5\% par an, doublement du nombre d'étudiants depuis 1991) avec de nouvelles structures et de nouveaux types d'étudiants. Il examine les politiques de l'enseignement supérieur dans toutes ses dimensions : gouvernance, financement, assurance de la qualité, équité, recherche et innovation, carrière universitaire, liens avec le marché du travail et internationalisation.

RENAUT Alain, Mettre les savoirs en culture : mission d'étude et de proposition sur la culture générale dans les formations universitaires, septembre 2004, 67 p. [rapport en ligne]

Ce rapport fait suite à un colloque (mai 2003 à la Sorbonne) qui réunissait des intervenants issus de différents horizons disciplinaires et géographiques. Il reprend les contenus des différentes séances. Dans la première, le contenu même de culture générale est précisé. Les deux séances suivantes établissent un état des lieux des expériences déjà en cours en France (universités de lettres et droit, universités des sciences et technologies) et à l'étranger. La quatrième séance a pour sujet la culture générale et la professionnalisation. Alain Renaut, en conclusion, émet un certain nombre de propositions et perspectives pour remédier au niveau insuffisant des étudiants en matière de culture générale.

SHATTOCK Michael, FULLER Steve, BARNETT Ronald et al., «L'entrepreneuriat», Politiques et gestion de l'enseignement supérieur, 2005, vol. 17, $n^{\circ} 3$, $151 \mathrm{p}$.

Le concept d'université entrepreneuriale a été le thème d'une conférence organisée par l'OCDE en 2000. Ce thème a continué à occuper une place centrale en Europe et dans le monde. Ce numéro spécial rassemble des études produites en amont d'une recherche sur ce sujet concernant une trentaine d'universités. Les trois premiers articles donnent le cadre théorique, suivi d'un aperçu sur les publications traitant de la culture entrepreneuriale et sur les politiques nationales favorisant celle-ci.

\section{TAYLOR Peter dir., Higher Education in the world : New Challenges and Emerging Roles for Human and Social Development, Palgrave MacMillan 2008, $272 p$.}

Le troisième rapport du Global University Network for Innovation (GUNI) s'intéresse au rôle que doit jouer l'enseignement supérieur au service du développement social et humain dans le contexte de la mondialisation. Après le point de vue de plusieurs organismes internationaux, la première partie analyse les défis que doit relever l'enseignement supérieur en réfléchissant à l'objectif éducatif, aux curricula, au rôle de la recherche, à l'engagement civique et aux réformes institutionnelles des établissements. La deuxième partie illustre ce rôle de l'enseignement supérieur à travers des exemples nationaux et régionaux.

UNESCO, Sciences sociales et humaines: stratégies et actions, 2004, 68 p. [rapport en ligne]

Le document apporte une vue d'ensemble du travail de l'UNESCO dans le domaine des sciences sociales et humaines. Ses activités sont concentrées autour de quatre domaines prioritaires : éthique des sciences et des technologies; défenses des droits de l'homme; prospective, philosophie et sciences humaines, sécurité humaine, paix et réconciliation; transformations sociales; programme anti-pauvreté. 


\section{SÉLECTION DE SITES INTERNET}

\section{CISS : Conseil international des sciences sociales (CISS)}

Créé en 1952, cet organisme bénéficie de l'appui de l'UNESCO. Il fédère un réseau d'associations, d'organisations et de conseils spécialisés en sciences sociales et en sciences du comportement à l'échelle mondiale, régionale et nationale. Le site est en anglais. http : //www. unesco. org/ngo/isscl

\section{EUA : European University Association}

L'association des universités européennes est le principal réseau universitaire en Europe (plus de 800 membres dans 46 pays). L'association est née en 2001 de la fusion de l'ancienne «association des universités européennes» et de la "confédération des Conférences de présidents d'université». Elle publie sur son site de nombreux rapports et documents concernant les politiques universitaires en Europe. Le site est en anglais. http://www.eua.be/about-eua/

\section{GUNI : Global university network for innovation}

Réseau mondial dont plus de 140 membres représentent les cinq continents. Il a été créé en 1999 par l'UNESCO, l'Université des Nations-Unies (UNU) et l'Université polytechnique de Catalogne (UPC) qui assure le secrétariat et le suivi de l'accord signé lors de la Conférence mondiale sur l'enseignement supérieur (CMES) qui s'est réunie à Paris en 1998. Le site est en anglais. http://www.guni-rmies.net/

\section{HAL-SHS (Hyper article en ligne - Sciences de l'homme et de la société)}

Bibliothèque numérique recevant et diffusant les productions intellectuelles de la recherche dans les disciplines des sciences humaines et sociales. HAL-SHS est une archive ouverte de publications scientifiques dont le fonctionnement, en particulier le contrôle des dépôts, est placé sous la responsabilité du Service d'Ingénierie Documentaire de l'ISH (Institut des Sciences de l'Homme, Lyon). http://halshs.archives-ouvertes.fr/

\section{IAU : Association internationale des universités}

Organisation affiliée à l'UNESCO, elle a été créée en 1950 pour encourager la coopération entre établissements d'enseignement supérieur dans le monde entier. Elle rassemble des universités membres de quelque 150 pays. http://www.unesco.org/iau/fre/index.html

\section{IMHE : Programme sur la gestion des établissements d'enseignement supérieur}

Forum international de l'OCDE destiné aux établissements de l'enseignement supérieur. On trouve sur le site un résumé des publications, des études de cas et les archives de leur bulletin d'informations IMHE-infos. http://www.oecd.org/

\section{Observatoire européen des politiques universitaires}

L'Observatoire européen des politiques universitaires a été créé en septembre 2004 à l'Université de Paris-Sorbonne (Paris-IV), à l'initiative d'Alain Renaut et avec le soutien de la Direction de l'enseignement supérieur. http://www.oepu.paris-sorbonne.fr/ 